\title{
Generation and Control of Turbulences in a Wind Tunnel
}

\section{América Torres, Roberto Tapia, Antonio Ramos}

División de Estudios de Posgrado, Facultad de Ingeniería Eléctrica, Universidad Michoacana de San Nicolás de Hidalgo, Morelia, México Email: atorres@dep.fie.umich.mx,rtsanchez@dep.fie.umich.mx,arpaz@umich.mx

How to cite this paper: Torres, A., Tapia, R. and Ramos, A. (2016) Generation and Control of Turbulences in a Wind Tunnel. Open Journal of Fluid Dynamics, 6, 453 471. http://dx.doi.org/10.4236/ojfd.2016.64033

Received: October 13, 2016

Accepted: December 26, 2016

Published: December 29, 2016

Copyright $\odot 2016$ by author and Scientific Research Publishing Inc. This work is licensed under the Creative Commons Attribution International License (CC BY 4.0).

http://creativecommons.org/licenses/by/4.0/

\begin{abstract}
In this paper, the generation and control of turbulences in a wind tunnel, for design, analysis and testing of test objects are proposed. Modifications to the wind tunnel are made in order to generate the turbulences in the test chamber. Specifically, for the turbulence analysis, the reflective symmetry concept is used in order to group the different turbulences cases generated in the wind tunnel test chamber. The Ansys software is used in order to model and analyze the wind tunnel. Specific platform or module known as Fluent is used for the analysis of the turbulence generated in the wind tunnel. To design proposal of a wind tunnel capable to generate and controlling turbulences is exposed in this paper. For this, the controller design and verification are performed by means of simulations. To obtain the control law structure, the inversion concept is used. The proposed control law is validated via a co-simulation implemented in the Simplorer module, with the aim of combining the power electronics part, controller and engine, with the proposed model wind tunnel.
\end{abstract}

\section{Keywords}

Wind Tunnel, Turbulence, Control

\section{Introduction}

A wind tunnel is a tool used in the investigation of the impact of the wind on solid objects real or to scale. Commercial wind tunnels get wind flow uniform rectilinear at a certain speed in the test chamber. The test chamber of the wind tunnel is where it places the objects, to observe the actual effect that the wind exerts on them, then they can be assessed or be designed solutions that may be necessary in the object of test. Therefore, a wind tunnel is suitable for aeronautical tests, in civil engineering, architectural designs, in the design of wind turbines, in sports training, etc. [1]. 
The first wind tunnel, known as "aerodynamic tube", was designed and built by Tsiolkovskyin 1897 [2].

On the other hand, Osborne Reynolds showed that the pattern of the wind flow on a scale object would be the same for the actual model if the Reynolds number for the flow out is the same in both cases. The Reynolds number is the central scientific justification for the use of scale models in the wind tunnels to simulate the real phenomena [2].

The Wright brothers used a simple aerodynamic tunnel in 1901, to study the effects of wind flow to pass through several shapes, while they were developed its "Wright Flyer", which was the first flying machine with engine and recorded the first sustained flight in an aircraft heavier than air.

The wind tunnel used by the Germans in the Second World War, used natural caves that when were excavated it, they increased its size and after, they are sealed in order to save large volumes of air that might be re-routed through the tunnels. This allowed the development research in Germany for the first supersonic wind tunnel, with a power of 100,000 horses of steam. After the Second World War, it was dismantled and moved to the United States [3].

Currently, there are different classifications of wind tunnels according to the air circulation and by its interior flow rate i.e. closed circuit, open circuit, subsonic, hypersonic (closed or open), among others.

Some recent researches about the wind tunnel are the next: in [4] the authors use the computational fluid dynamics (CFD) to study the effect of the wind on buildings, as well as in [5], the numerical modeling of flow and dispersion on various construction forms in a wind tunnel using the CFD is presented. Also, in [6] the CFD is used to simulate the natural environment of a road vehicle in a closed climatic wind tunnel.

On the other hand, in [7] the authors present a combined study between experimental and computational performance of the aerodynamics in a small vertical axis wind turbine, where the experimental tests are performed in a wind tunnel with laminar flow in the test chamber. In [8] is analyzed the wind turbines in a virtual wind tunnel made in CFD; however, the authors did not model turbulences. Additionally, it has been developed specific articles of wind tunnel designs, as for example: In [9], is presented the design of components and geometric arrangements of the wind tunnel from analytical procedures based on the requirements set out in this article, and then the Ansys Fluent software is used in order to verify the obtained results.

The design and use of the wind tunnel it has been made and published in many articles, only a few references have been mentioned here. However, the design of a wind tunnel is directly related to its application. Then, in this concept this article is focused in the design of a wind tunnel that it can be able to generate laminar and turbulent flow in its test chamber, and control it. This is made in order to provide a better analysis in the objects of test, for example it can be made a vibrational analysis in a wind turbine (scaled model) in order to develop a better material for the blades or to develop a different control law that could be capable to mitigate these oscillations.

The innovation of this article is focused in a specific tunnel design that permits the 
generation and control of turbulences for scale models of residential wind turbines (low power). The analysis presented in this article will allow the physical construction and instrumentation of it, in order to test different wind turbine design.

This article is organized as follows: In Section 2, the wind tunnel characteristic and components are presented. In Section 3, the turbulences generation is exposed. Section 4 presents the Ansys implementation. A turbulences analysis is made in Section 5. Section 6 presents 2 case studies for the turbulences generation. The turbulences control law is developed in Section 7. In Section 8, the proposed control law is validated through a Co-simulation. Finally, in Section 9, the conclusions and future work of this investigation are made.

\section{Wind Tunnel}

The open circuit wind tunnel with a closed test chamber is chosen for the design of the wind tunnel, due to this configuration has a lower construction cost, it is easier to introduce some smoke class to display the flow, and is not continued its operation. In addition, it is a subsonic wind tunnel type, because the maximum speed considered in the test chamber is $20 \mathrm{~m} / \mathrm{s}$.

At first, the design of the wind tunnel is made as a classical one; this means that the wind tunnel is designed for having a laminar flow in the test chamber.

The total length of the wind tunnel is shown in Figure 1, as well as the elements that compose it: inlet area (fan), test chamber, flow conditioning mesh, areas of contraction and dissemination (diffuser).

The test chamber is rectangular with $0.80 \mathrm{~m} \times 0.80 \mathrm{~m}$ dimensions, and is chosen by its simplicity to fabricate it, is easy to fit and remove the model, and the low losses coefficient.

The area of contraction is used to increase the speed of the air. This speed increase is based in the Venturi effect, which said: If the flow of a fluid is constant but the section decreases the speed increases, also if the kinetic energy increases, energy determined by

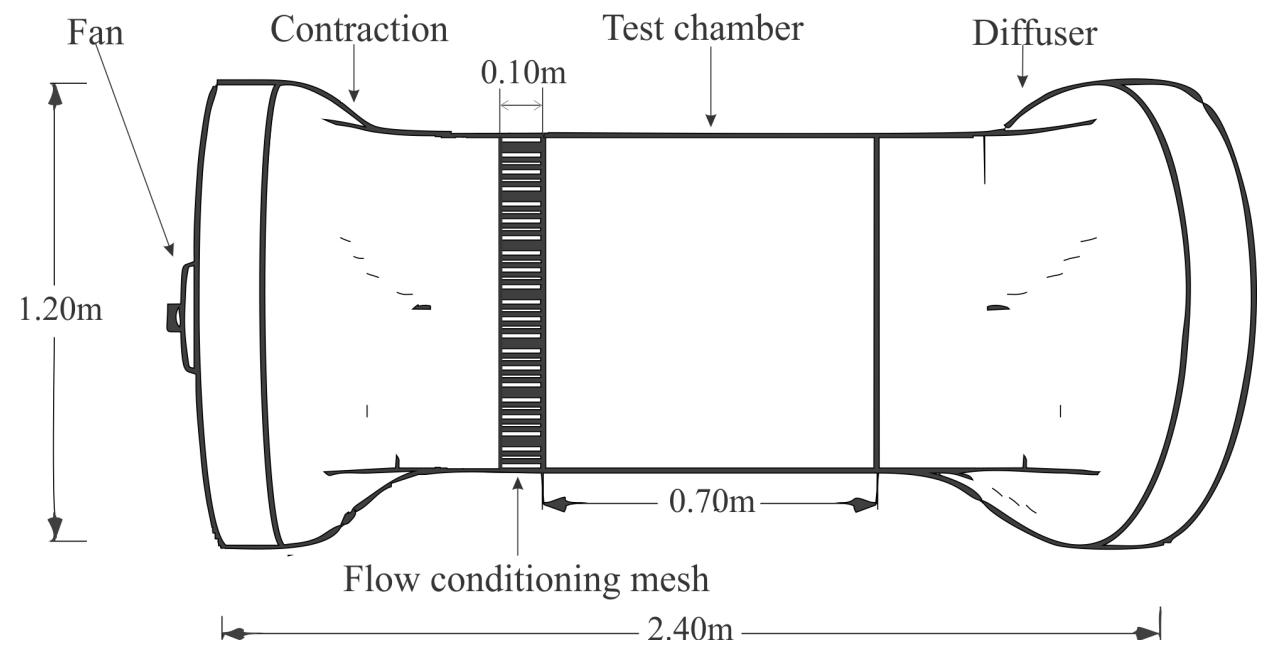

Figure 1. Wind tunnel. 
the value of the pressure decreases [10].

For the mesh conditioner flow it is chosen a hexagonal structure of the interior cells, representing the best choice in terms of configuration due to its lowest coefficient of losses flow ( $k=0.20$, where $k$ is the losses coefficient).

\section{Turbulences Generation}

\subsection{Turbulence Principles}

The mechanical turbulence concept is used in this article in order to generate the turbulence in the test chamber. The mechanical turbulence alters the shear layer allowing to generate the turbulence. Some examples of mechanical turbulences, are those that are caused by the passage of air on buildings, trees, hills, etc. (see Figure 2), can also be generated by the difference in the speed of the wind or in its direction between two points, this phenomenon is known as shearing [11].

The shear layer is a region very thin, with large gradients of speed. The speeds in both sides of the separation surface are different, giving rise to a thin region where the speed changes quickly. These shear layers can be idealized as surfaces of discontinuity in the speed [12].

Figure 3 shows the shear layer formed between two speeds of different magnitude that follow the same direction (Figure 3(a)), and also the formed it between two speeds of the same intensity, but with opposite directions (Figure 3(b)).

The shear layer concept is explained in the Figure 4. It is assumed a surface of discontinuity where the speeds on both sides of the discontinuity are equal and opposite. When it is applied a small disturbance (which generates a light undulation as shown in Figure 4) it will increase the subtly fluid speed on the convex surface parties (A, B', C, $\left.D^{\prime}\right)$, and it will slightly decrease on the concave surface parties (A', B, C', D) [12].

By considering a stationary flow and applying the Bernoulli equation (to calculate the load value of pressure, load of lifting and shifting of the burden of speed, through circulate fluid system [13], it has to be a net force of pressure acts amplifying the shocks, so the shear layer is unstable and tends to wrap. The wave begins to distort more and more with the time, eventually becoming vortices.

The formation of a whirlwind is associated with a large Reynolds number (considered greater than 4000), if the viscosity effects are negligible. Otherwise, for the case of

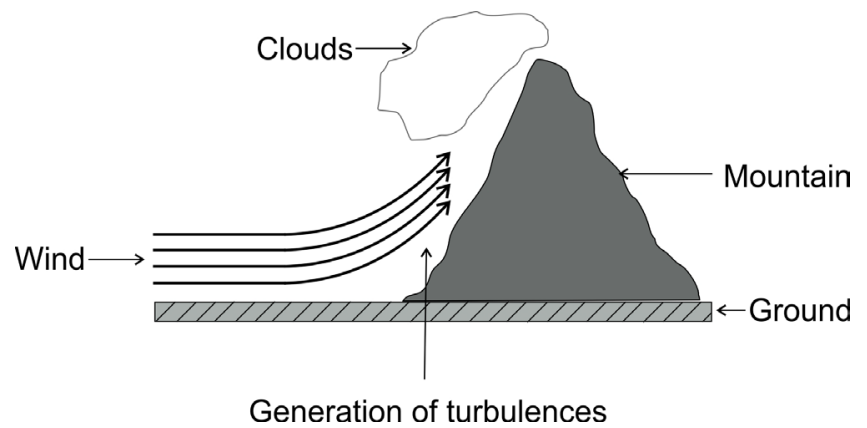

Figure 2. Mechanical turbulence. 


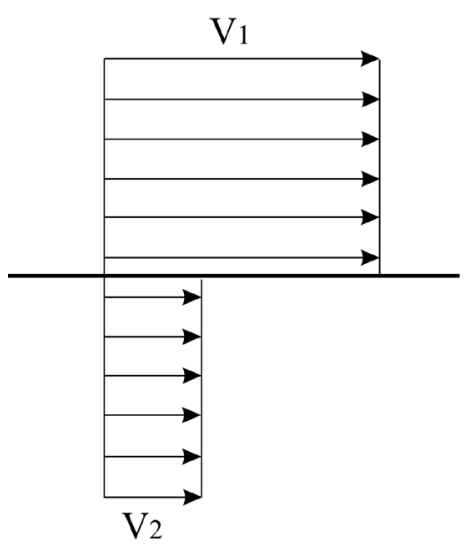

(a)

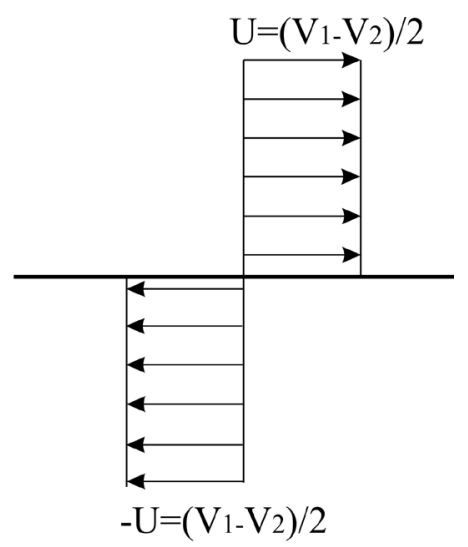

(b)

Figure 3. Shear layer (a) Different magnitude, same direction, (b) Opposite direction, same speed.

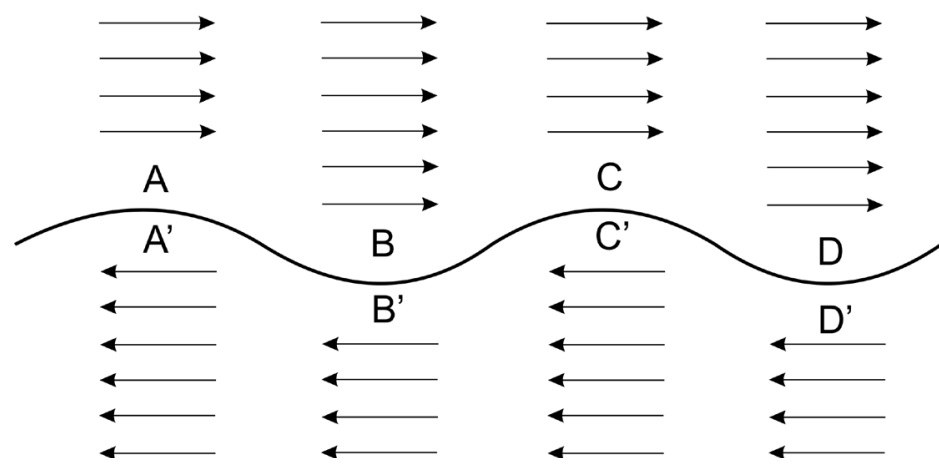

Figure 4. Slights disturbance.

a low Reynolds numbers (considered less than 2000), the viscosity influences and tends to dissipate or stabilize the discontinuity. In Figure 5 it is shown the representation of an instability with the whirlwinds clearly defined (Figure 5 upper part), and a turbulence fully developed (Figure 5 bottom) [13].

\subsection{Wind Tunnel Turbulences Generation}

In the wind tunnel design, it is proposed to use the concept of mechanical turbulence, in order to alter the shear layer, either with flows of different magnitudes or directions. To achieve this, 3 fans are added on the top and bottom of the test chamber with a diameter of $0.168 \mathrm{~m}$, respectively (see Figure 6). These fans allow generating a vertical flow inside the test chamber. In addition, in the input wind tunnel are placed 3 fans, instead of one. These fans allow generating a horizontal flow for the shear layer. This allows having different zones inside the wind tunnel (whit different flow intensity), and also allows to alter the shear layer in different regions inside the test chamber. Figure 6 shows the wind tunnel modifications.

Figure 6 also shows the nomenclature used in order to identify each fan of the wind tunnel. 


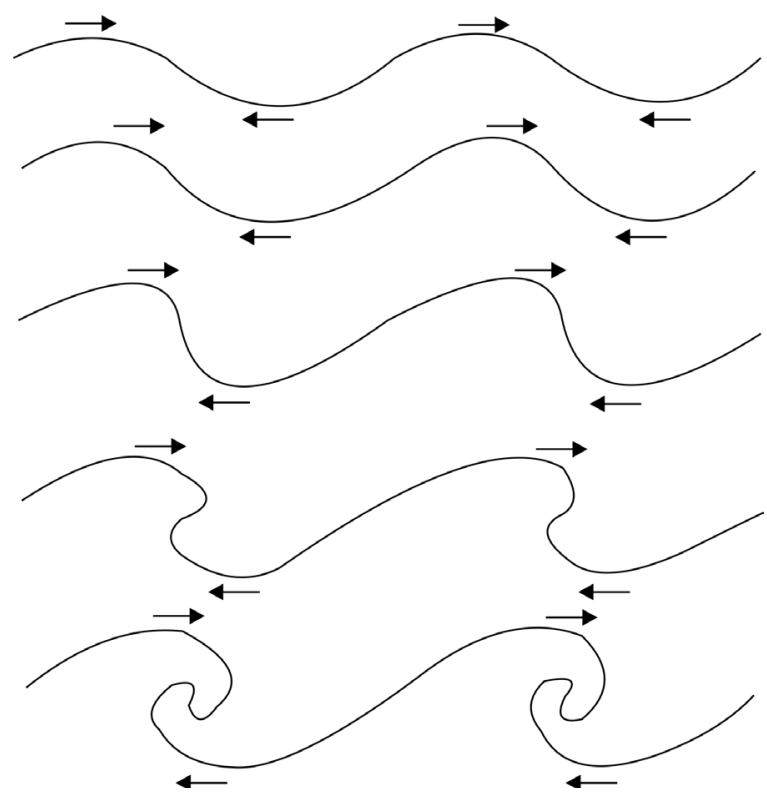

Figure 5. Formation of instability in the shear layer.

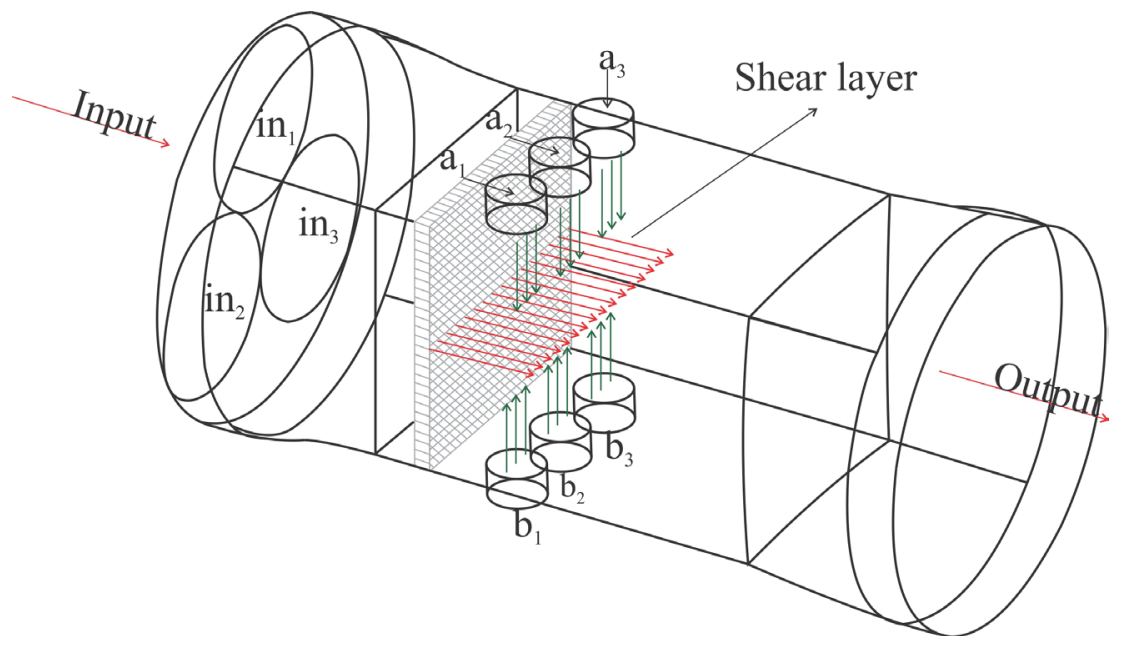

Figure 6. Turbulence generation in the wind tunnel.

As the wind tunnel is used to test scale models, the six fans added in the test chamber allows having three different zones (vertically), inside the test chamber. In these three zones, the scale model can be analyzed for different attack angles. Then, it will be three zones for affect and analyze the structure (three angles of attack in the model). An angle of attack is an angle that forms the string line of the object with the direction of the speed of the air. By changing the angle of attack it can change the characteristics of the soil bearing capacity, which is a force caused by the fluid in a direction perpendicular to the direction of movement of the body.

\section{Ansys Fluent Simulation}

To analyze the behavior of the flow inside the wind tunnel through the test chamber, 
the Ansys Fluent [14] is used in order to simulate it.

This program approximates the solution of the flow behavior by means of finite volumes. Then, the results depend on the number of elements used in the mesh. In addition, ten numbers of elements are improved by having a mesh with the smallest number of asymmetrical elements. Figure 7 presents the generated mesh for the wind tunnel model of Figure 6.

The chosen type of mesh is unstructured and contains 1,178,608 elements. Due to Fluent approximates the solution, it is important to determine the quality of the mesh. This is measured with the asymmetry tool (Skewness), which allows identifying changes in the elements of the model with regard to the original items. In the histogram of Figure 8, they are shown the numbers of items, as well as the quality of the mesh measured with the tool "Mesh Metric".

By observing Figure 8, it is concluded that the mesh quality is good, as the majority of the elements (of which it is composed) are within the range [0,0.5], and only 35,300 elements have a poor quality, due to the fact that they are at the maximum allowed (0.95). The classification of the elements is based on Table 1.

The Ansys Fluent simulation conditions are the next:

- Solver: Pressure based type, transient time, formulation absolute velocity.

- Solution Methods:

o Pressure Velocity Coupling: Coupled Scheme.

o Spatial discretization: Least Squares Cell Based for the Gradient, Second Order for

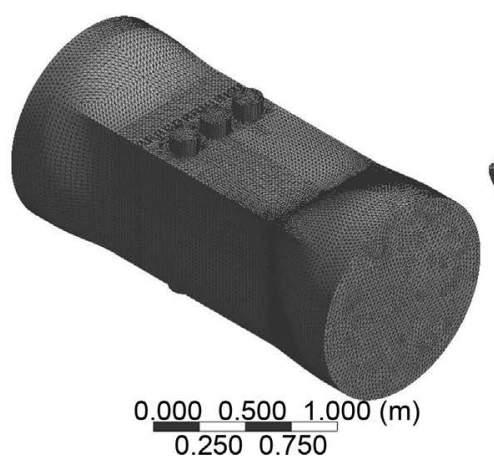

(a)

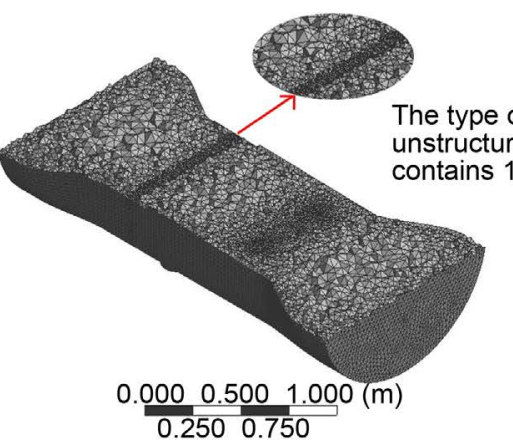

(b)

Figure 7. Wind tunnel mesh: (a) Isometric view, (b) longitudinal cutting.

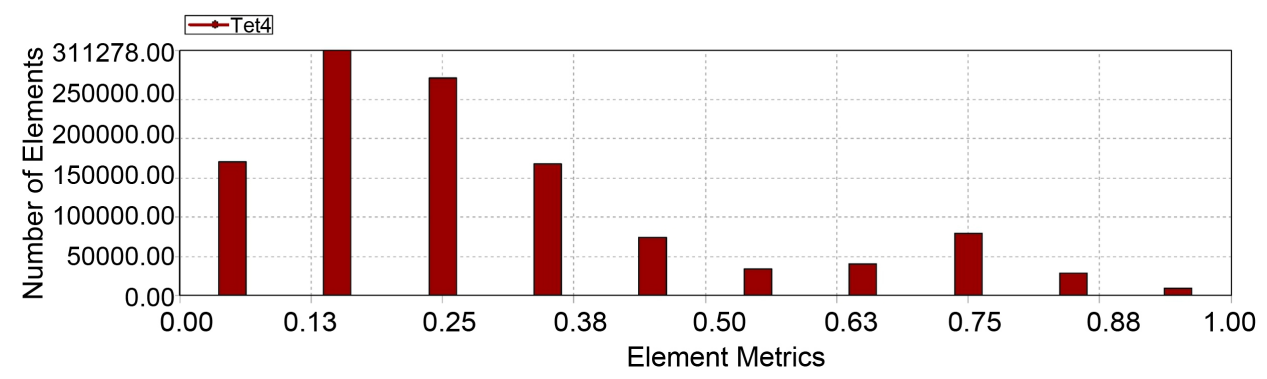

Figure 8. Wind tunnel meshing quality. 
Table 1. Range of skewness values and the corresponding cell quality [14].

\begin{tabular}{cc}
\hline Skewness value & Quality cell \\
\hline 0.9 to $<1$ & Degenerate \\
0.75 to 0.9 & Bad (silver) \\
0.5 to 0.75 & Poor \\
0.25 to 0.5 & Fair \\
$>0$ to 0.25 & Good \\
0 & Excellent \\
\hline
\end{tabular}

the Pressure, Second Order Upwind for the Momentum.

The results of the post-processing of Fluent are shown in Figure 9.

In Figure 9(a) it is shown the representation of the wind tunnel, with the 3 fans of input turned on at a speed of $20 \mathrm{~m} / \mathrm{s}$, in which it is noted that the speed in the test chamber is uniform with an approximate value of $25 \mathrm{~m} / \mathrm{s}$.

In Figure 9(b) are activated all the fans of the wind tunnel (3 in the input tunnel, and 6 in the test chamber). This figure also shows a not uniform speed inside the test chamber. Then, it is achieved alter the cut layer getting an unstable area in the test chamber $30 \mathrm{~m} / \mathrm{s}$.

\section{Turbulence Generation Analysis}

Taking in to account that there have been added three fans in the wind tunnel design (see Figure 6), in order to generate the turbulences inside the test chamber, different fans can be turned ON or turned OFF in different time instants. Then, to identify the fans activation, the uses of a binary representation is proposed. Thus, the use of 0 represents that the fan is turned OFF (deactivated), and the use of 1 represents that the fans in turned ON (activated).

They are nine fans, three for each region of the tunnel (input, test chamber upper and lower), then each region has $2^{n}$ possible combinations, where $n$ is the number of fans. Then, it is necessary to identify the fans assets both on entry and in the test chamber. As it was shown in Figure 6, the fans are distributed in three principal regions: the tunnel input, and the top and bottom in the test chamber. Every region has three fan, and then in order to identify which fan is activated, one number formed by three digits will be used (i.e. 1-1-1). The first digits of this number correspond to the input fan, and its value is equal to 1 when the $i n_{1}=1, i n_{2}=0$ and $i n_{3}=0$, this means that fan $i n_{1}$ is activated while fan $i n_{2}$ and $i n_{3}$ are deactivated. The number is equal to 2 when the $i n_{1}=0, i n_{2}=1$ and $i n_{3}=0$, this means that fan $i n_{2}$ is activated while fan $i n_{1}$ and $i n_{3}$ are deactivated. In this way the binary combination is used in order to have 8 possible values (from 0 to 7 ) in this first digit. For the other two regions same consideration is taken; the top and bottom region correspond to the second and third digits, respectively. As an example, the 1-1-1 combination means that fan $i n_{1}, a_{1}$ and $b_{1}$ are 


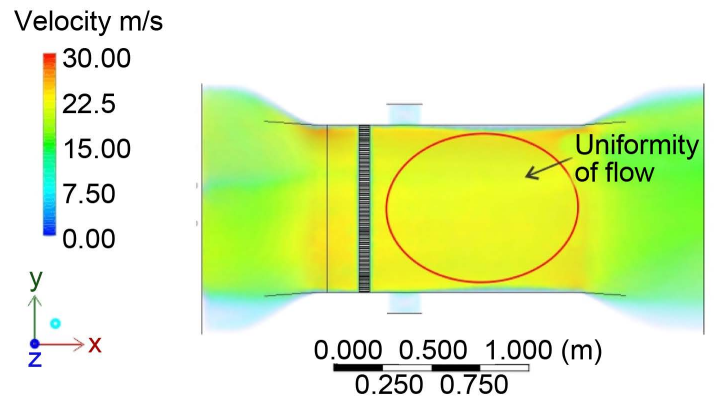

(a)

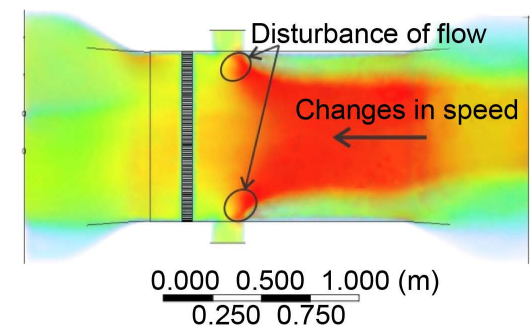

(b)

Figure 9. (a) 3 fans turned on input tunnel, (b) 6 fans turned on test chamber and 3 fans turned on input tunnel.

activates ( $i n_{1}=1, a_{1}=1, b_{1}=1$ ), while the rest of fan are deactivated.

The flow behavior analysis for different combinations is performed by using the reflective symmetry concept. This concept is defined as: a transformation with respect to a plane of symmetry (flat surface that divides the object in two equal parts), in which each point of a figure is associated to another point called image, which meets the following conditions [15]:

1) The distance of a point and its image to the plane of symmetry is the same.

2) The segment joining a point with its image, it is perpendicular to the plane of symmetry.

The plane of symmetry is defined as the flat surface that divide the object in two halves exactly equal (see Figure 10), and the center of symmetry are imaginary points located in the interior of the object, in which pass the main lines and planes of symmetry [16]. Figure 10 shows some examples of the reflective symmetry.

It is proposed to have two symmetrical planes (horizontal and vertical) between two or more combinations to compare the flow in the test chamber. For this, they are located inside the test chamber 5 air flow sensors; each of them is placed in a horizontal symmetric plane, and one in the symmetry center. In Figure 11(a) are shown the 5 sensors placed inside the test chamber.

Figure 11 shows the imaginary cuts made in the test chamber with the objective of finding symmetry in the flow behavior for different combinations in the activation of the fans. Figure 11 is highlighting the horizontal and vertical symmetrical flat cuts in the test chamber center; as well as the symmetry center.

The objective to identify the symmetrical planes is made in order to group the generated turbulences in symmetrical areas. This allows classifying different combination of the fan activations and using it ensemble with the control to generate and control the turbulence in the desired test chamber zone.

As mentioned before, different combinations are possible because 9 fans are placed in the wind tunnel. Then, in order to classify the different combinations, each of them it is called a symmetry case. The symmetry cases are obtained by modifying the activation/ deactivation of the input fans, which it is indicated by the first digit of the combinations, 

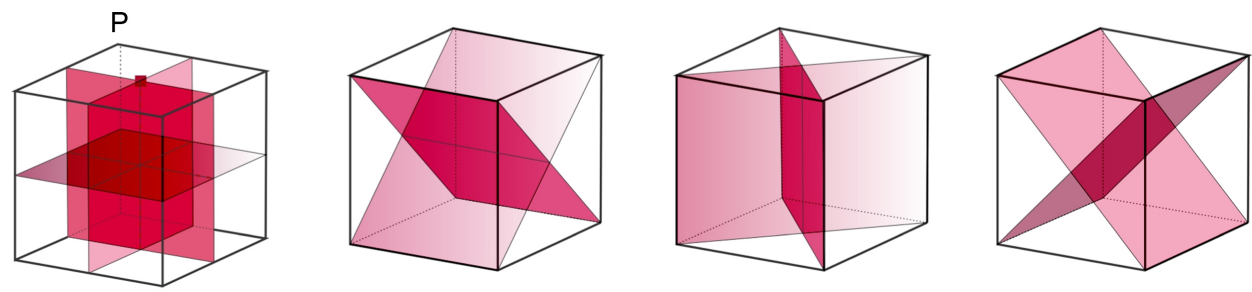

Figure 10. Wind tunnel meshing quality.

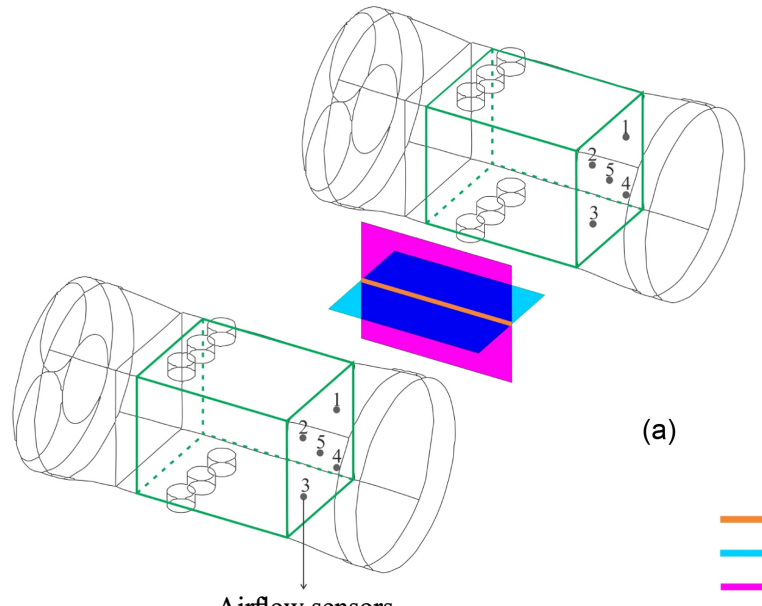

Center of symmetry

Symmetrical horizontal plane

Airflow sensors

Symmetrical vertical plane

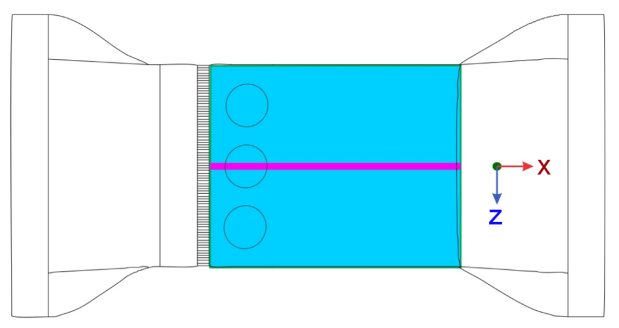

(b)

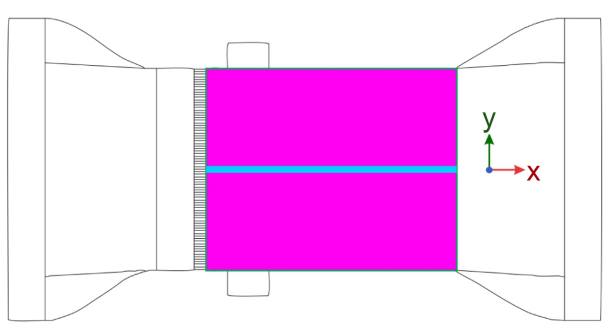

(c)

Figure 11. Virtual division test chamber. (a) Isometric view, (b) top view, and (c) left side view.

followed by the second and third digits that indicates the activation/deactivation of the top and lower fans in the test chamber, respectively.

The symmetric combinations are grouped in 24 symmetry cases, which are shown in Table 2.

In Table 2, for example, the case 1 indicates that the combination 1-1-1 is symmetrical to the combination 2-4-4. This means that if turbulence would be created in the zone which cover the combination 1-1-1, this turbulence can also be created in the symmetrical region (inside the test chamber) if the combination 2-4-4 is used.

\section{Case Study}

In this section is analyzed one case among the 24 possible (Table 2). The rest of cases can be easily analyzed likewise.

Considering the case 4 shown in Table 2, the Figure 12 shows the possible views for 
Table 2. Symmetrical cases.

\begin{tabular}{cccccc}
\hline Case & \multicolumn{2}{c}{ Combinations } & \multicolumn{2}{c}{ Case } & \multicolumn{2}{c}{ Combinations } \\
\hline 1 & $1-1-1$ & $2-4-4$ & 13 & $1-1-4$ & $2-4-1$ \\
2 & $1-3-3$ & $2-6-6$ & 14 & $1-4-1$ & $2-1-4$ \\
3 & $1-6-6$ & $2-3-3$ & 15 & $1-3-6$ & $2-6-3$ \\
4 & $3-1-1$ & $3-4-4$ & 16 & $1-6-3$ & $2-3-6$ \\
5 & $3-3-3$ & $3-6-6$ & 17 & $3-1-4$ & $3-4-1$ \\
6 & $4-1-1$ & $4-4-4$ & 18 & $3-3-6$ & $3-6-3$ \\
7 & $4-3-3$ & $4-6-6$ & 19 & $4-3-6$ & $4-6-3$ \\
8 & $5-1-1$ & $6-4-4$ & 20 & $5-1-4$ & $6-4-1$ \\
9 & $5-3-3$ & $6-6-6$ & 21 & $5-4-1$ & $6-1-4$ \\
10 & $5-4-4$ & $6-1-1$ & 22 & $5-3-6$ & $6-6-3$ \\
11 & $7-1-1$ & $7-4-4$ & 23 & $5-6-3$ & $6-3-6$ \\
12 & $7-3-3$ & $7-6-6$ & 24 & $7-1-4$ & $7-4-1$ \\
\hline
\end{tabular}

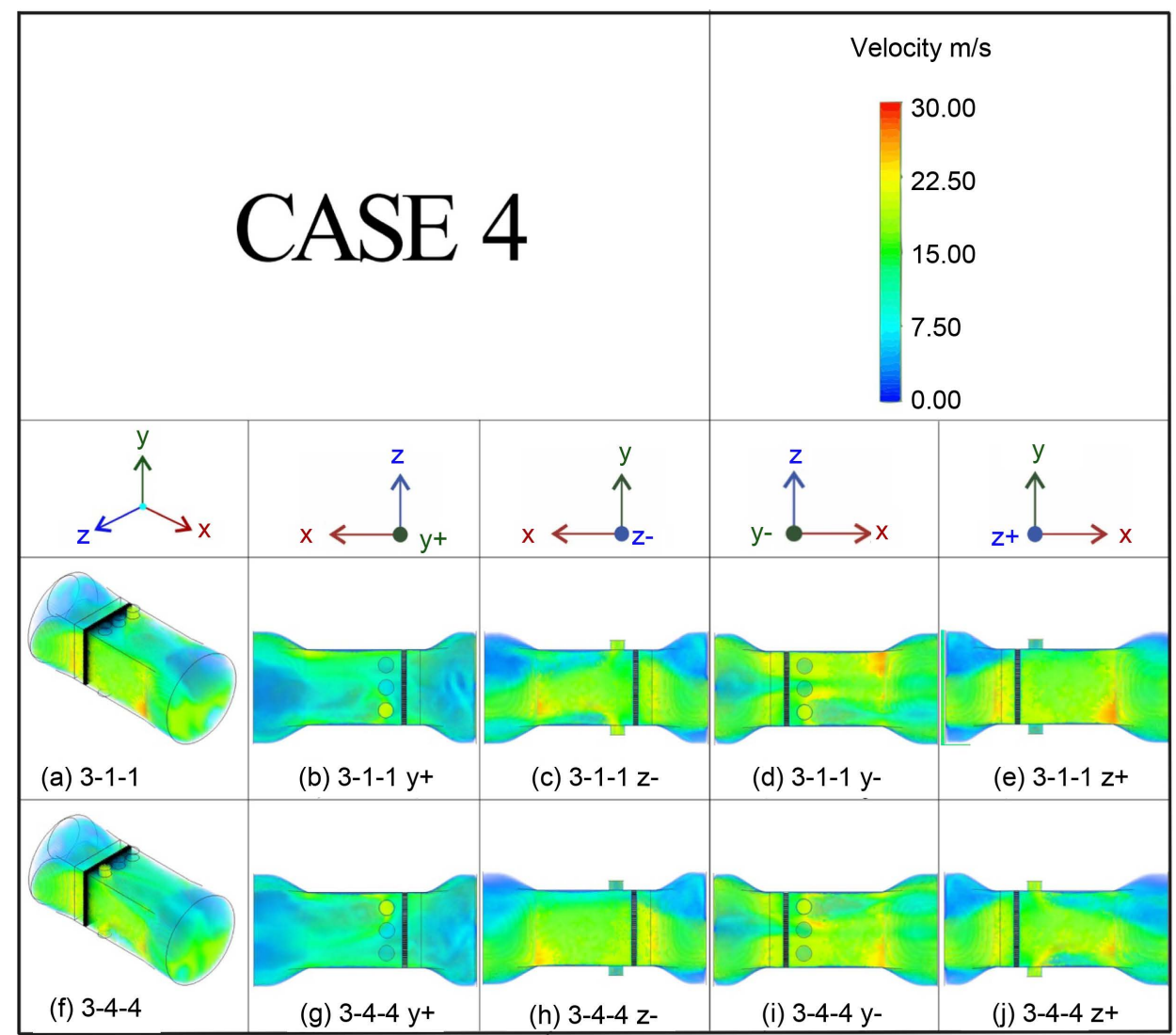

Figure 12. Case study-Case 4.

this case, which is formed by the symmetric combinations 3-1-1 and 3-4-4. The figures that visually have symmetry in the vertical plane in the flow behavior are: Figure 12(b) 
with the Figure 12(g), and also the Figure 12(d) with the Figure 12(i). On the other hand, figures that show a horizontal symmetry in the flow behavior are: The Figure 12(c) and the Figure 12(j), as well as comparing Figure 12(e) and Figure 12(h).

To examine the behavior of the flow inside the wind tunnel, it is observed that the flow is directed toward the bottom of the test chamber, either on the right side (Figure 12(d)) or left (Figure 12(i)), depending on the combination). It also presents an alteration of lateral flow because they are assets the fans in the test chamber. For example, for combination 3-1-1 are active a_3 and b_3 and the flow is altered in the right side (see Figure 12(c)), otherwise for combination 3-4-4 are active $a \_1$ and $b \_1$ and the flow is altered in left side (see Figure 12(j)). In addition, it can be seen in Figure 12(e) and Figure $12(\mathrm{~h})$ that there is a uniform flow, because on that side there are no fans turned on in the test chamber.

Figure 13 shows the isometric perspective for both combinations, as well as the symmetry that share. If is required to generate a turbulence oriented toward the right or left side of the test chamber without flow alteration in the opposite side (for right side see Figure 12(c) and Figure 12(e), and for left side see Figure 12(j) and Figure 12(h)), and with a flow behavior in the bottom of the test chamber (oriented toward the left (see Figure 12(j)) or right side (see Figure 12(c)) it is necessary to choose either of the two combinations, only taking into account in which area is required.

The wind speed in the test chamber in both combinations of Figure 13 has a maximum speed of $25 \mathrm{~m} / \mathrm{s}$, approximately, and the minimum speed is near to $17 \mathrm{~m} / \mathrm{s}$. The speed increases (when it is compared to case 1) because two fans are turned ON at the tunnel input ( $a_{2}$ and $a_{3}$ ), as a consequence, there is a greater concentration of flow in the test chamber. In addition, in the side of the test chamber where the fans are turned OFF, it has the highest speed and not disturb the shear layer.

\section{Turbulence Control}

The fan addition in the test chamber allowed generating the turbulences inside the wind tunnel. Then, in order to control these turbulences, it is necessary to add the

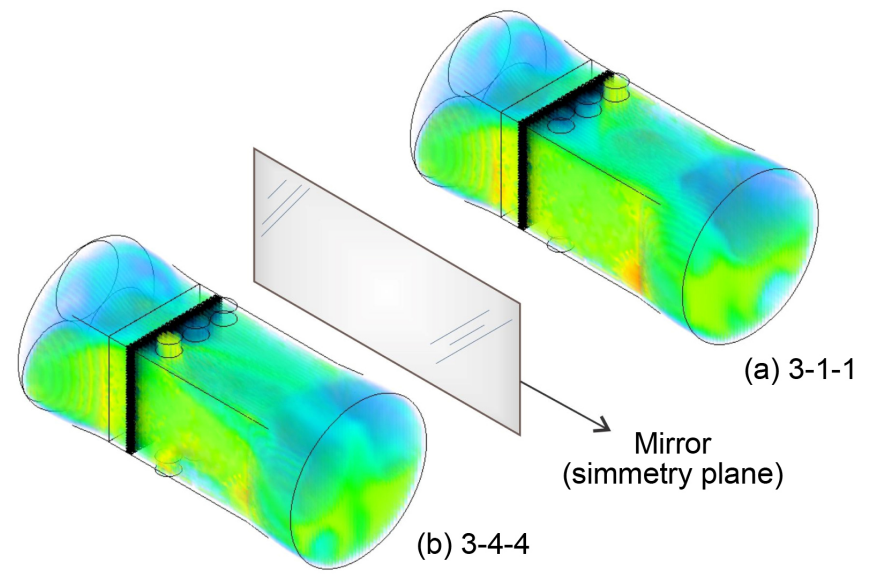

Figure 13. Case study—Case 4: isometric view. 
drivers and sensors. Figure 14 shows the block diagram used to perform the turbulences control.

The AC-DC converter (commonly called rectifier converter) makes the conversion that provides the voltage and current required to drive the DC motor (fan). The $\mathrm{H}$ bridge is used to control the fan speed with the motor rotation, and thus keep constant the chosen speed. The speed sensor is required to measure the speed of the wind inside the test chamber, and thus to be able to regulate the speed of the DC motor. Some sensors are located inside the test chamber as it was show in Figure 11(a). The sensors also allow identifying the generated turbulence. The control structure showed in Figure 14 it is used for each fan located in the wind tunnel.

As it is considered a DC motor for each fan of the wind tunnel, in order to obtain their control law, a mathematical representation is needed. The Equation (1) represents the equation of a simplified motor, which is a first-order differential equation for the angular speed.

$$
V_{a}(t)=\frac{J R_{a}}{k_{a}} \dot{\omega}(t)+\left(\frac{R_{a} f}{k_{a}}+k_{b}\right) \omega(t)
$$

where $V_{a}(t)$ is the input voltage of the electrical part, $J$ is the inertial moment, $R_{a}$ is the motor resistance, $k_{a}$ is the couple constant, $k_{b}$ is the counter-electromotive force constant, $f$ is the viscose friction coefficient, and $\omega(t)$ is the angular velocity.

Appling the Laplace Transform in Equation (1), it can be obtained the transfer function of the angular velocity in relation to the input voltage in the DC motor, as follows:

$$
G_{\dot{\theta}}(t)=\frac{\omega(s)}{V_{a}(s)}=\frac{1}{\frac{J R_{a}}{k_{a}} s+\left(\frac{R_{a} f}{k_{a}}+k_{b}\right)}
$$

To formulate the control law, it is used the inverse system concept [17]. The chosen variable to control is the angular velocity $\omega(t)$ in the DC motor, which is taken in the frequency domain of Equation (2), thus the obtained reverse system represents the open loop control. As it is well kwon, a structure in open loop does not allow a good dynamics regulation. Then, in order to obtain the close loop control law, it is imposed the dynamic of error $\dot{\varepsilon}+C \varepsilon=0$, where $C$ represents the controller to be chosen, and the error is defined as $\varepsilon=\left(\omega_{\text {ref }}-\omega\right)$. These expressions are replaced in Equation (1) to obtain the Equation (3).

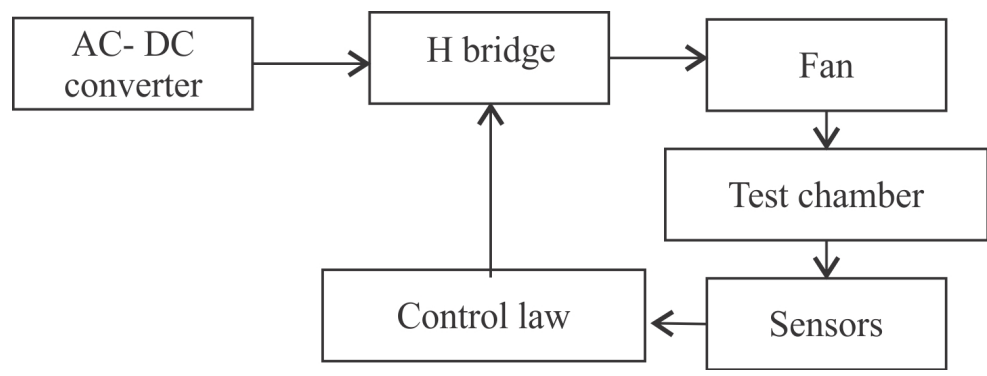

Figure 14. Structure of the control law. 


$$
V_{a}(s)=B \omega(s)+A s \omega_{\text {ref }}(s)+A C \varepsilon(s)
$$

where: $A=J R_{a} / k_{a}, B=\left(R_{a} F / k_{a}+k_{b}\right)$

Figure 15 represents the control blocks diagram in the frequency domain, $\tilde{A}$ and $\tilde{B}$ correspond to the implicitly parameters that appear in the control law, and they are considered an approximation of the real one ( $A$ y $B$ from Equation (3)).

It is important to notice that the structure of the control law makes appear two feed-back loops, one is the traditional feed-back (which regulate the reference error), and the other one is directly link whit the sensed angular velocity. Also and fed-forward loop with a derivative action it is shown in the structure. This loop appears due to the fact that the inverse model has been used in order to obtain the control. In practice, the derivative actions are not desired because the signal noise can introduce a considerable error in their calculation. Nevertheless, as constant signals are used in the system (as reference), this action cannot have a considerable impact in the system, only it will be present when a suddenly change is made on reference signal. Then the impact of this fed-forward loop is present only in the transients. The Transfer function in closed loop is given by:

$$
\frac{\omega(s)}{\omega_{\text {ref }}(s)}=\frac{\tilde{A} s+C(s) \tilde{A}}{A s+B-\tilde{B}+C(s)}
$$

For the Equation (4) it is chosen a proportional + integral controller (PI), this means that $C(s)=k_{p}+k_{i} / s$. The selection of a PI controller is performed because it uses the simplified equation of the DC motor, allowing to have a first order transfer function. If the estimated values in Equation (4) exactly match the values of the system, the reference is followed perfectly. The above would be an ideal case, but in practice it is common that the parameters present some variations or differences, however, it has been demonstrated that the control structure is robust for a parametric variation values [18]. The strength that presents the structure of control for this specific case allows the control of the DC machine to be successful, even when the model of the wind tunnel is not known and represents a disturbance in the system.

\section{Co-Simulation in Simplorer}

The concept of co-simulation is used in order to verify the proposed design and control

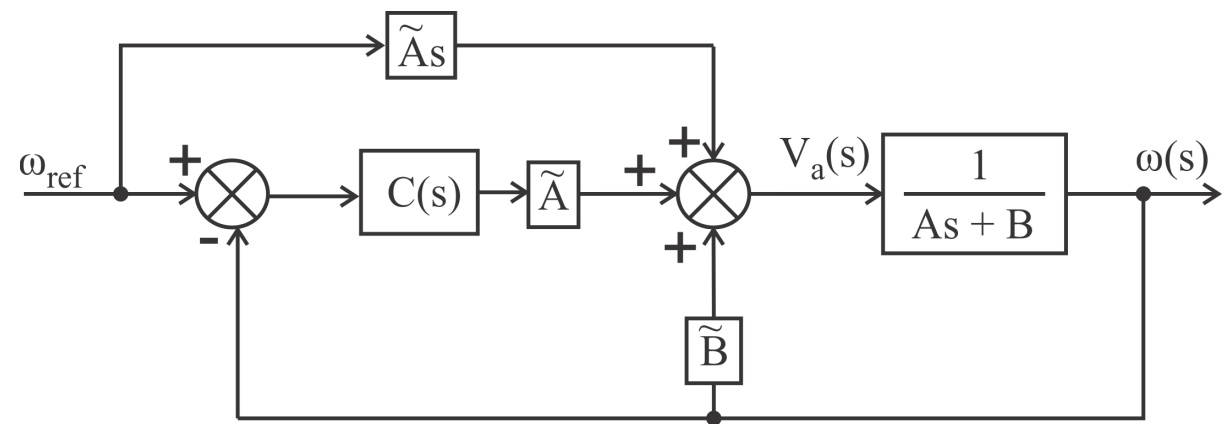

Figure 15. Block diagram of the control law. 
law in the wind tunnel.

The co-simulation combines, in one simulator, the wind tunnel model (developed in Fluent) and its control, the AC-DC converter and the H bridge (implemented in Simplorer). The control law developed before is applied in closed loop, considering the wind tunnel model as an output perturbation in the system (DC motor). Figure 16 shows the block diagram of the co-simulation.

Figure 16 shows the interaction between two modules of Ansys: Fluent (plant) and Simplorer (control law). Simplorer represents the output of a data series that are the result of the co-simulation. The shared variable is the wind speed, which is monitored in Fluent and controlled by Simplorer. Fluent has objects (sensors) that monitor the variable of the wind speed.

The co-simulation in Simplorer is performed taking into account the next scenario:

- Starts the co-simulation with all input tunnel fans turned ON, during 1 second with a $20 \mathrm{~m} / \mathrm{s}$ wind speed.

- At the instant equal to 1 second, some input tunnel fans are turned OFF. The corresponding fans that are turned OFF are selected in order to perform the corresponding combination (required turbulence).

- At instant equal to 2 seconds, the test chamber fans required to perform the combination in course are turned $\mathrm{ON}$ in order to alter the flow wind behavior (generate the turbulence).

This scenario is used in the case study, as it is presented in the next subsection.

\section{Case Study-Co-Simulation}

The co-simulation of case 4 (case study) verifies the behavior of the control law, as well as shows the symmetry that exists in the combinations of this case (see Table 2).

Figure 17 shows the co-simulation results. As mentioned before, the scenario starts with a wind speed of $20 \mathrm{~m} / \mathrm{s}$ in the three input wind tunnel fans, until the second equal to one. After this, it has been turned OFF the $i_{1}$ fan, in both combinations (3-1-1 and 3-4-4), called FLUENT1 and FLUENT2, respectively (see Figure 17(a)). In addition, in Figure 17(a) is observed that the $i n_{2}$ and $i n_{3}$ fans in both combinations varies around the $20 \mathrm{~m} / \mathrm{s}$ speed, in order to maintain the desired speed in the test chamber. On the other hand, in Figure 17(b) is observed at the instant equal to 2 seconds that $a_{3}$ and $b_{3}$

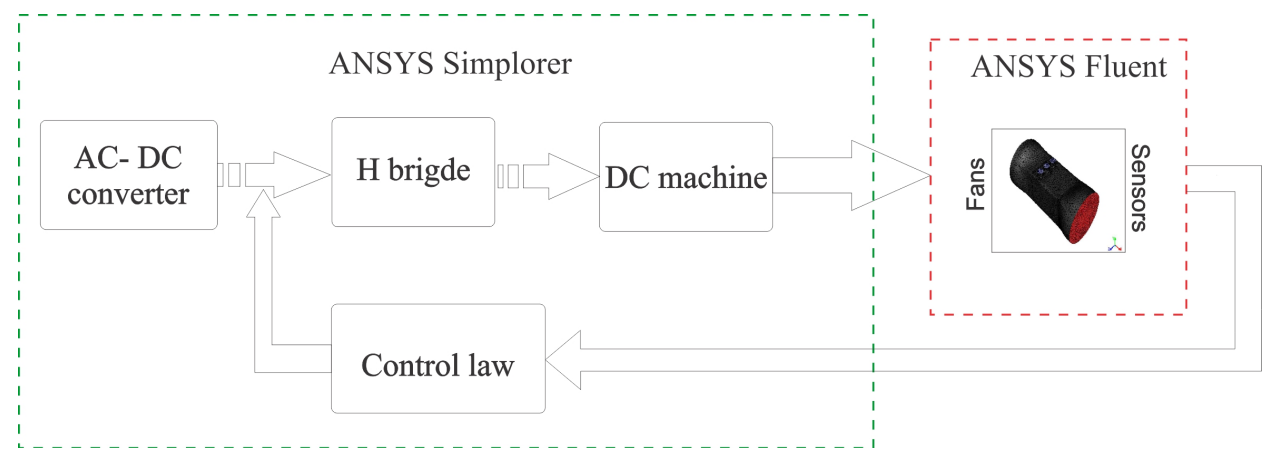

Figure 16. Block diagram of the Co-simulation in Simplorer. 


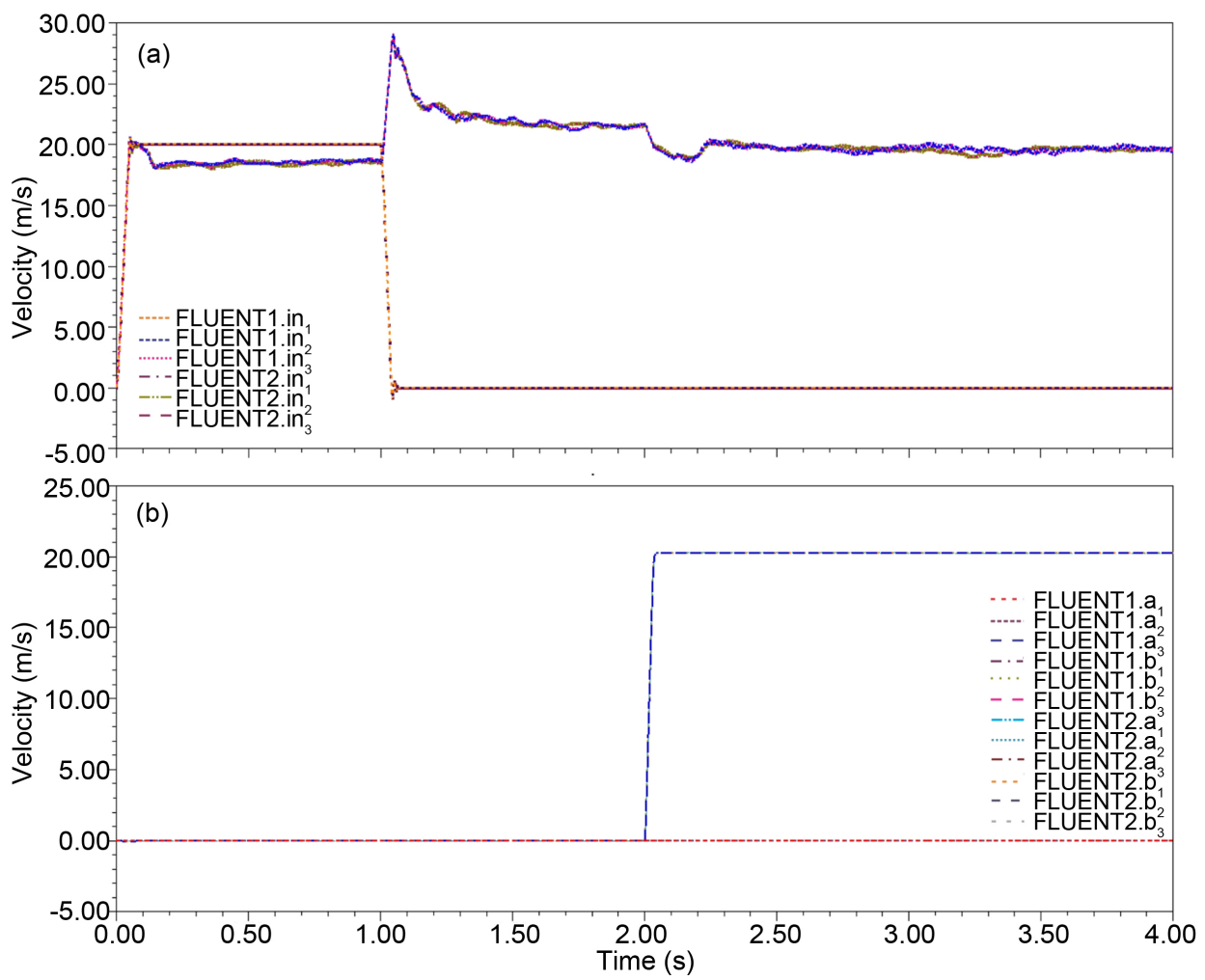

Figure 17. Case study-Case 4: (a) Input fans, (b) Tests chamber fans.

fans for FLUENT1 and $a_{1}$ and $b_{1}$ fans for FLUENT2 are turned ON in order to generate the required turbulence on the test chamber. These fans have a speed of $20 \mathrm{~m} / \mathrm{s}$.

It is important to mention that curves in Figure 17(a) shown very high values in the transitory at 2 seconds. This is because these forms are taken from the input fluent model and corresponds to the output controller, and then these changes are necessaries in order to maintain the desired speed wind inside the test chamber.

Figure 18 shows the sensors (in the test chamber) responses. Figure 18(a) shows that the sensor that best describes the wind behavior is the sensor 4 for the combination 3-1-1 (FLUENT1), measuring a $20 \mathrm{~m} / \mathrm{s}$ speed with variations of $\pm 0.1 \mathrm{~m} / \mathrm{s}$. For the Figure 18(b) the sensor 3 of the combination 3-4-4 (FLUENT2) is the one that best describes the wind behavior to maintain a $20 \mathrm{~m} / \mathrm{s}$ speed approximately. By comparing the Figure 18(a) and Figure 18(b), it is observed that the sensor 5 has smaller instant changes in comparison with the other sensors, measuring a speed of $17.5 \mathrm{~m} / \mathrm{s}$ with variations of $\pm 1 \mathrm{~m} / \mathrm{s}$, this is because this is the sensor placed in the symmetry center (wind tunnel center inside the test chamber) where less wind disturbances are detected and presented in this combination. This is because they have been turned ON 2 fans of the input wind tunnel, so there is a greater flow concentration in the test chamber.

Also in Figure 18(a) and Figure 18(b) is shown that the sensors that measure the lower speed are: sensor 2 for the combination 3-1-1 (FLUENT1.sen ${ }_{2}$ ) and sensor 1 for the combination 3-4-4 (FLUENT2.sen $)_{1}$ ). This is because the $a_{3}$ and $b_{3}$ (combination 3-1-1) and, $a_{1}$ and $b_{1}$ (combination 3-4-4) fans are turned $\mathrm{ON}$ in the test chamber, which 

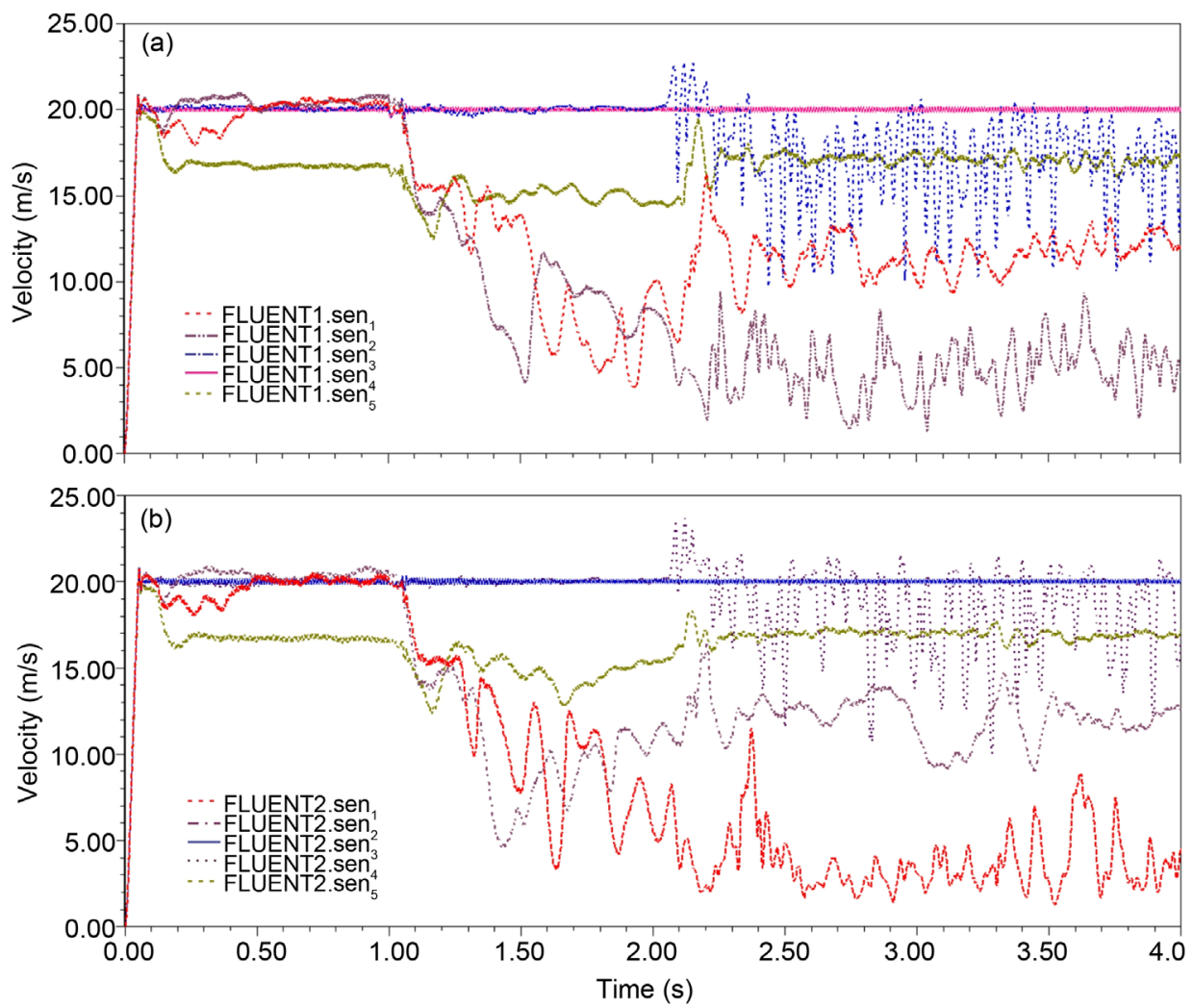

Figure 18. Case study_Case 4: (a) Sensors of combination 3-1-1, (b) Sensors of combination 3-4-4.

means that the flow behavior in that area is altered.

A comparison of similar sensor in both combinations is needed. Then, Figure 19 compares the sensor 4 (combination 3-1-1) with the sensor 3 (combination 3-4-4), in order to analyzing the existing symmetry for this case. It can be seen in Figure 19(a) that the sensors responses are equals; their speed value is around $20 \mathrm{~m} / \mathrm{s}$. In Figure 19(b) is shown a zoom of these sensors, and it can be seen that the speed measurement in the sensors fluctuates for sensor 4 (combination 3-1-1) from 19.81 to $20.16 \mathrm{~m} / \mathrm{s}$, while for the sensor 3 (combination 3-4-4) from 19.85 to $20.13 \mathrm{~m} / \mathrm{s}$. These results shows the case 4 symmetry, which generates a turbulence toward the bottom of the test chamber (see $Y$ - plane), and with a right lateral flow behavior ( $Z+$ plane) combination 3-1-1 or left (Z- plane) combination 3-4-4 (all this in Figure 12).

It is important to mention that although there are differences in the results obtained (asymmetric elements), the turbulence is generated within the area that had been planned for each of the combinations; this allows you to control the region in which it is generated by the turbulence when a scale model is placed in the test chamber.

Finally, with the results obtained from the simulation of this case, it is checked that the control law developed in Section 7, performs the function for which it was designed.

\section{Conclusions}

In this paper, the generation and control of turbulences in the test chamber of a wind 

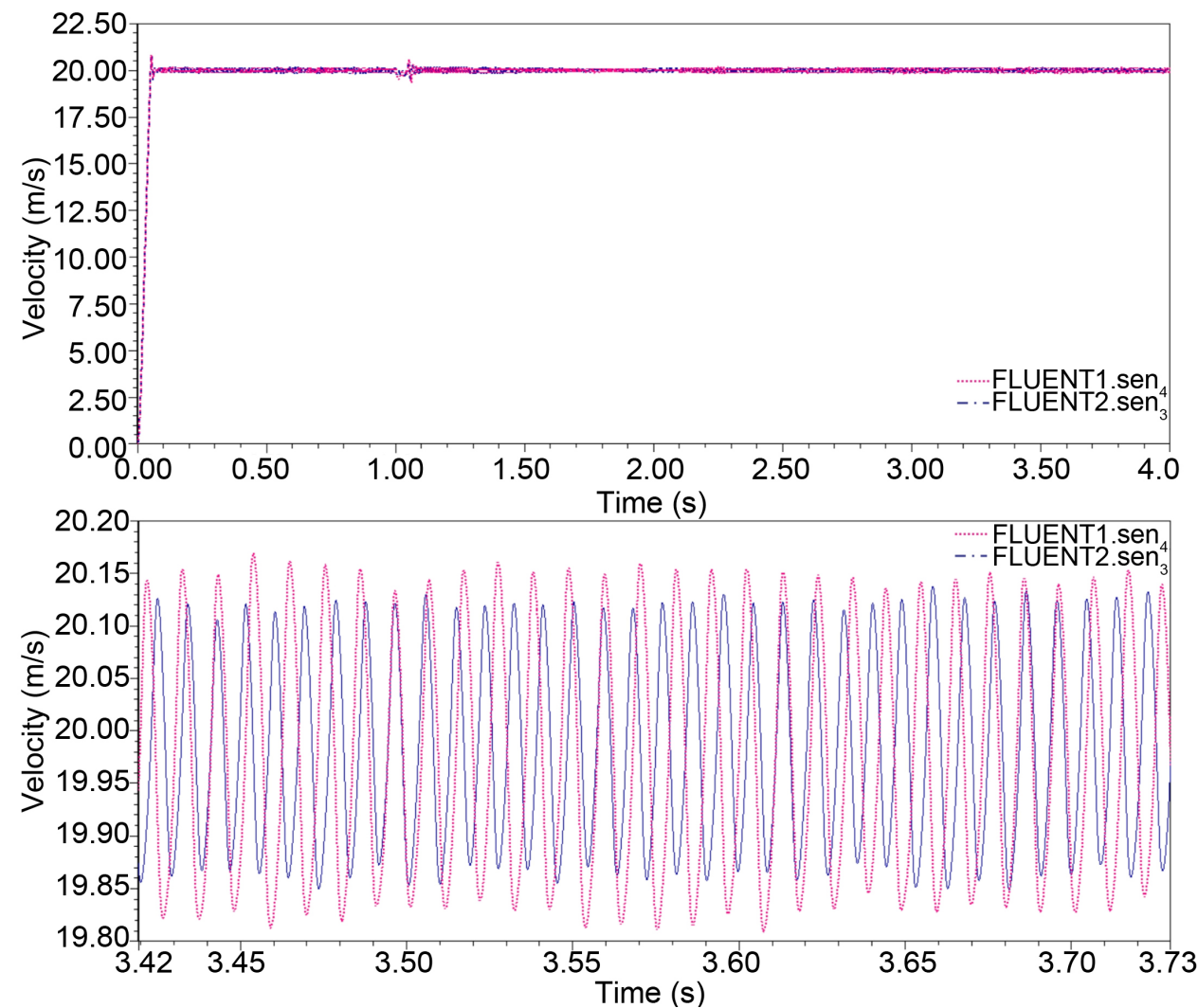

Figure 19. Case study-Case 4: Comparison sensor 4 (3-1-1) with sensor 3 (3-4-4), (a) Disturb of the shear layer, (b) Zoom of the comparison.

tunnel was proposed. The design of a wind tunnel has been made in order to generate the turbulences.

To simulate the wind tunnel, the Ansys Fluent software is used, which allows to analyze the wind flow behavior in the test chamber, in order to identify the generated turbulence.

For the generation of turbulence inside the test chamber of the wind tunnel, the mechanical turbulence concept has been used.

A control was proposed in order to generate the turbulence. For this, the concept of inverse control was used. The different elements of the system were presented, as for example, the DC motor for modeling the fans, as well as an H-bridge to speed up or slow down the DC motors by means of its rotation.

The concept of co-simulation was used to validate the proposed model and control. The results showed with a case study allowed showing the performance of the control. The other cases that have been shown in Table 2 maybe verified in the same way; this article presented only one case.

\section{Acknowledgements}

The authors thank the support given by the Secretaria de Educación Pública under the project UMSN-PTC-378. 


\section{References}

[1] ITER (2011) Instituto Tecnológico y de Energías Renovables S.A.

[2] Barlow, J.B., Pope, A. and Rae, W. (1999) Low Speed Wind Tunnel Testing. John Wiley \& Sons, Inc., Hoboken.

[3] Cook, N.J. (1978) Determination of the Model Scale Factor in Wind-Tunnel Simulations of the Adiabatic Atmospheric Boundary Layer. Journal of Wind Engineering and Industrial Aerodynamics, 2, 311-321. https://doi.org/10.1016/0167-6105(78)90016-8

[4] Chang, C.-H. and Meroney, R.N. (2003) The Effect of Surroundings with Different Separation Distances on Surface Pressures on Low-rise Buildings. Journal of Wind Engineering and Industrial Aerodynamics, 91, 1039-1050. https://doi.org/10.1016/S0167-6105(03)00051-5

[5] Meroney, R.N., Leitl, B.M., Rafailidis, S. and Schatzmann, M. (1999) Wind-Tunnel and Numerical Modeling of Flow and Dispersion about Several Building Shapes. Journal of Wind Engineering and Industrial Aerodynamics, 81, 333-345. https://doi.org/10.1016/S0167-6105(99)00028-8

[6] Ghani, A., Aroussi, A. and Rice, E. (2001) Simulation of Road Vehicle Natural Environment in a Climatic Wind Tunnel. Simulation Practice and Theory, 8, 359-375. https://doi.org/10.1016/S0928-4869(00)00028-8

[7] Howell, R., Qin, N., Edwards, J. and Durrani, N. (2010) Wind Tunnel and Numerical Study of a Small Vertical Axis Wind Turbine. Renewable Energy, 35, 412-422. https://doi.org/10.1016/j.renene.2009.07.025

[8] Sumner, J., Sibuet Watters, C. and Masson, C. (2010) CFD in Wind Energy: The Virtual, Multiscale Wind Tunnel. Energies, 3, 989-1013. https://doi.org/10.3390/en3050989

[9] Caro Rincón, F., Fernández López, S., Lora Ibarra, D. and Ruiz Sánchez, J. (2012) Diseño de un Túnel de Viento Subsónico para Hélices de Máximo 30 pulgadas. Master Thesis, Universidad de Buenaventura, Bogota.

[10] Streeter, V.L. (1992) Mecánica de los Fluidos. 4th Edition, McGraw-Hill, México, D.F., 13-37.

[11] Sendiña Nadal, I. and Pérez Muñuzuri, V. (2006) Fundamentos de Metereología. Universidad de Santiago de Compostela, Compostela, España, 24-31.

[12] Meseguer, J., Sanz, J.L., Ortega, F. and Chiappe, L. (2008) Control de Capa Límite en el Vuelo a Bajos Números de Reynolds. Ingeniería Aeronáutica y Astronáutica, 15-24.

[13] Mott, R.L. (2006) Mecánica de Fluidos. 6th Edition, Pearson Educación México.

[14] ANSYS (2010) Introduction to ANSYS FLUENT. http://www.ansys.com/

[15] Wald, R.M. (1984) General Relativity. University of Chicago Press, Chicago. https://doi.org/10.7208/chicago/9780226870373.001.0001

[16] Girbau, J. (1993) Geometria Diferencial iRelatividat.Universidad Autònoma de Barcelona, Barcelona, 153-180.

[17] Mukherjee, A. and Karmakar, R. (2000) Modelling and Simulation of Engineering Systems through Bond Graphs. Alpha Science International Ltd, 353-357.

[18] Sánchez, R., Dauphin-Tanguy, G., Guillaud, X. and Colas, F. (2010) Bond Graph Based Control of a Three Phase Inverter with LC Filter Connection to Passive and Active Loads. Simulation Modelling Practice and Theory, 18, 1185-1198. https://doi.org/10.1016/j.simpat.2010.05.016 
Submit or recommend next manuscript to SCIRP and we will provide best service for you:

Accepting pre-submission inquiries through Email, Facebook, LinkedIn, Twitter, etc. A wide selection of journals (inclusive of 9 subjects, more than 200 journals)

Providing 24-hour high-quality service

User-friendly online submission system

Fair and swift peer-review system

Efficient typesetting and proofreading procedure

Display of the result of downloads and visits, as well as the number of cited articles

Maximum dissemination of your research work

Submit your manuscript at: http://papersubmission.scirp.org/

Or contact ojfd@scirp.org 